\title{
Use of molecular markers and major genes in the genetic improvement of livestock
}

\author{
Hugo H. Montaldo ${ }^{1}$ \\ Division of Animal Science, University of New England, Armidale, NSW. \\ 2351. A ustralia. \\ Tel : 61-2-6773-3004, Fax : 61-2-6773-3275 \\ E-mail:hmontald@metz.une.edu.au. \\ Cesar A. Meza-Herrera \\ Unidad Regional Universitaria de Zonas Aridas. Universidad Autónoma Chapingo. A.P. No. 8. \\ Bermejillo, Durango. México. 35230 \\ E-mail:cmeza@chapingo.uruza.edu.mx \\ *Corresponding author
}

Recent developments in molecular biology and statistics have opened the possibility of identifying and using genomic variation and major genes for the genetic improvement of livestock. Information concerning the basis of these techniques and their application to the genetic improvement of animals is reviewed. Main marker molecular marker systems in animals (RFPL and microsatellites), genome maps, methods for detecting marker-QTL linkages and use of marker assisted selection, genetic fingerprinting and mixture models based on segregation analysis are analyzed. The characteristics in where the application of marker assisted selection can be more effective are those that are expressed late in the life of the animal, or controlled by a few pairs of genes. The first example corresponds to the longevity and carcass characteristics in meat producing animals, the second, to the resistance to certain diseases or defects of simple inheritance. The detection of major genes using mixture models with segregation analysis can direct the work of identification of DNA marker genotypes towards populations and characteristics with greater probability of detecting a QTL. The present trend indicates that molecular, pedigree and phenotypic information will be integrated in the future through mixture models of segregation analysis that might contain QTL effects through the markers, polygenic inheritance and uses powerful and flexible methods of estimation such as Gibbs Sampling.

During the last five decades, the application of methods based on population genetics and statistics allowed the development of animals with a high productive efficiency. These systems are based on simplified models of genic action that assume a large number of or genes with small individual effects in the expression of the phenotype (polygenes) and emphasizes the average genic effects (additive effects) over ther interactions. The basis is predicting the breeding values of the animals using phenotypic and genealogical information. Properties of the predictions are equivalent to the levels of correlated random effects of a mixed linear model or best linear unbiased predictors (BLUP) which is based to a large extent on the work of $\mathrm{C}$. Henderson (Henderson, 1984).

Important advances to some of the economically important characters in several species of livestock has been achieved based on phenotypic performance, however, several limitations of these methods of improvement based on population genetics alone are becoming evident with time. Their efficiency decreases when the characteristics are difficult to measure or have a low heritability. Additionally, selection has been generally limited to those characteristics that can properly be measured in large numbers of animals. Some characteristics such as the rate of survival are expressed very late in the life to serve as useful criteria of selection. Also, the traditional selection within populations has not been very efficient when the selection objective involves several characteristics with unfavorable genetic correlation, for example, milk production and protein content of milk (Schwerin et al., 1995).

Additional terms which are biologically plausible could be added to the model, but seldom their effects can be estimated accurately with only phenotypic and genealogical information in outbreed animal populations. These complications might indude interactions between genes in the same locus (dominance) or in different loci (epistasis) and between specific genes and the environment (genotype by environment interactions).

The use of molecular techniques could help to solve some of the limitations of the current methods. The capacity to generate dense genetic maps in each species can in principle allow the complete genome to be evaluated for quantitative trait loci (QTL) with a major effect on the phenotype. This information can then be used in genetic programs (Kinghorn et al.,1994). A lso, advances in the statistical and mathematical techniques applied to the modelling of the genetic processes are helping to make an optimal use of molecular and phenotypic information in the selection process. (Bovenhuis et al., 1997; Hoeschele et al., 1997).

Molecular techniques allow detecting variation or polymorphisms exists among individuals in the population for specific regions of the DNA. These polymorphisms can be used to build up genetic maps and to evaluate differences between markers in the expression of particular traits in a family that might indicate a direct effect of these differences in terms of genetic determination on the trait. More probably, the can prove some degree of linkage of the QTL effecting the trait and the marker (Albert et al., 1994; Lewin, 1994; Stein et al., 1996).

Recently, methods have been developed to detect the presence of major genes from the analysis of pedigreed data in absence of molecular information (Bovenhuis et al., 1997). These methods, based on mixture models and segregation analysis, allow to direct the potentially expensive and time consuming genotyping activities towards populations and characteristics with a greater probability of being controlled by a QTL and to optimize the collection of molecular data (Kinghorn et al., 1994).

The objective of this paper is to review some advances on molecular genetics and statistical methodology to identify and estimate the effects of major gene effects in animal populations, and its potential use in the genetic improvement of livestock. 


\section{Restriction fragment length polymorphisms}

Sax (1923) showed that an observable gene with simple mendelian inheritance could act as a marker for the segregation of a gene involved in the expression of a quantitative trait. Early work on detection of genome variation of potential markers focused on the analysis of proteins and blood type variation, but this was found to be impractical to be used as genome markers. Protein systems lack adequate polymorphism, genome coverage was low and there was a requirement for the gene to be expressed at the phenotypic level to make detection possible (Drinkwater and Hetzel, 1991).

Beginning in 1980, an accelerated understanding of the structure and function of the genome has been obtained as animals (Schimenti, 1998). At first, techniques were developed to visualize the differences at the level of the structure of the DNA based on the use of bacterial restriction enzymes that cut the DNA at sites with specific nucleotide sequences. With this basis, the technique denominated restriction fragment length polymorphisms (RFLPS) was developed. Theidentification of RFLPs requires the use of gel electrophoresis to separates the DNA fragments of differing sizes followed by transfer of the fragments to a nylon membrane (Southern blot) and visualization of specific DNA sequences using radioactive or chemiluminescent probes exposed to an X-ray film (Drinkwater and Hetzel, 1991).

This methodology was the standard method in the identification of RFLPs before 1986, situation that limited the identification of the whole genome variation in animals. This technique allowed the identification of only two alleles per locus and is slow to be used with large genome size in mammals, where about $3 \times 10^{9}$ individual nucleotides are present in the total DNA content (A lbert et al., 1994; Brash, 1994: Lewin, 1994; Wilmut et al., 1992). The reduced variability observed in domestic animals by inbreeding makes many RFLPs sites non-informative. Modification of the RFLPs technique is possible to identify multiple alleles, but still have practical drawbacks that make preferable the use of the PCR based microsatellite markers (Drinkwater and Hazel, 1991).

\section{Polymerase Chain Reaction (PCR)}

Mullis et al. (1986) developed the process known as polymerase chain reaction or PCR. This allows to the amplification or reproduction in great amounts of particular regions of the DNA. In order to initiate the process of replication of the DNA, two informing sequence codes denominated primers are required which promote the beginning and reversion of the reaction of the polymerase (RNA-pol) at particular locations of the genome. A reproduction or amplification of thousands of copies of a chromosomal region or gene of interest is obtained by repeated cycles of synthesis and denaturalization (chain separation) of the DNA using temperature changes. Since the primers are specific sequences to bond to a determined region of the DNA, only the specific amplification of the desired sequence of DNA instead of amplifying the DNA in its totality is obtained (Buratowski, 1994; Koleske and Young, 1995; Stein et al., 1996).

\section{Minisatellites}

Parallel to the development of the PCR, a new type of polymorphism of DNA also known as hypervariable minisatellites were discovered in the DNA structure. These minisatellites are defined regions of DNA with polymorphisms in the number of repeated nucleotide sequences of around $25 \mathrm{bp}$ in length. These minisatellites can be used for search for marker genes associations and as DNA fingerprints in paternity testing (Albert et al., 1994; Lewin, 1994; Bishop et al., 1995; Smith and Smith, 1993; Stein et al., 1996).

\section{Microsatellites}

Microsatellites systems are composed of DNA repeats in tandem at each locus. The tandem repeats are usually simple dinudeotides (such as (TG) n ) with each dinudeotide repeated about ten times. Its high degree of polymorphism in the number of repeats (n) allow its use as location markers in genome mapping. The length of each allele is determined by PCR using specific oligonudeotides primers flanking the repeated sequence. The DNA products are visualized after electrophoresis. PCR based Microsatellites techniques facilitated the construction of genome maps in most livestock species because its abundance in the genome, the specificity of the primers, its high degree of polymorphism which several alleles and their easy detection (Albert et al., 1994; Lewin, 1994; Bishop et al., 1995; Smith and Smith, 1993; Stein et al., 1996).

\section{Other marker systems}

Other types of DNA genetic markers used mainly in plant breeding but with potential use in animals are randomly amplified polymorphic DNA (RAPD), simple sequence repeats (SSR) and amplification fragment length polymorphisms (AFLP) (Specht, J. personal comunication, 1996).

New types of markers are single nudeotide polymorphic markers (SNP) based on high density DNA arrays (Chee et al., 1996 ). In this technique of "gene chips", DNA corresponding to thousand of genes are arranged on small matrices ("chips") and probed with labeled CDNA from a tissue of choice. The information is then read by a device to be downloaded to a computer (Schimenti, 1998).

\section{Sequence analysis}

Highest resolution of DNA variation can be obtained using sequence analysis. Sequence analysis provides the fundamental structure of gene systems. DNA sequencing is generally not practical to identify variation between animals for the whole genome, but is a vital tool in the analysis of gene structureand expression (Drinkwater and Hazel, 1991).

\section{Molecular genome maps}

A step towards the use of genomic information in livestock improvement is the location of all markers and protein coding genes in the chromosomes. Thus the development of genetic maps of the species of interest is required for detecting QTL using genetic markers (Bovenhuis et al., 1997). Mapping based on recombination frequencies could be built up on the basis of genetic marker genotyping from DNA obtained from populations of reference, with several collaborating laboratories worldwide (Beattie, 1994). Development of PCR based techniques and microsatellite markers, increased the efficiency of the mapping processes in all living organisms.

An aid in the development of livestock genome maps, has been the high level of conservation of gene sequences between humans, cattle, sheep, goat, pig and mice. By such reason, once loci of particular DNA sequences has been mapped in one species, the information is frequently of help in the genome mapping in another. Livestock species mapping had been greatly facilitated by the increasing availability of human and murine sequences (Beattie, 1994; Schimenti, 1998). 
Additionally, the construction of physical maps has been favored by means of the development of techniques like the hybrids of somatic cells and fluorescence in situ hybridization (FISH) (Beattie, 1994). With the development and use of these techniques, genome mapping has had a spectacular advance in the last years. Whereas in 1990150 positions in the bovine genome had been identified, for 1998 this number surpasses 1,500 positions.

Examples of current genome mapping information available in the Internet for several mammal species are:

http:/ / vega.crbm.cnrs-mop.fr/ Vert-Genome_DBS.html

http:/ / sol.marc.usda.gov/ genome/ cattle/ cattle.html

http:/ / sol.marc.usda.gov/ genome/ swine/ swine.html

http:/ / ws4.niai.affrc.go.jp/ dbsearch2/ mmap/

\section{Candidate gene approach}

There are two main categories of genomic information that can be used in genetic improvement of livestock:

1. Genes with known on the expression of certain protein.

2. Genes with effects detected on the characteristic in statistical terms. These markers are based on polymorphic sequences of the DNA that is in the same chromosome (linked) to a gene of quantitative effect (QTL.

Examples of the first type (1) in livestock are:

1.1 The gene associated with porcine malignant hyperthermia. In this case, a (C T) mutation at position 1843 of the gene calcium release channel ryanodyne receptor locus $(R y r l)$ in recessive condition have a high probability of being the cause of the susceptibility to this disease (Huges and Lowden, 1998).

1.2 The myostatin $(M H)$ locus which contains a series of mutations which disrupt the myostatin function, detected in several European cattle breeds causing muscular hypertrophy (Georges et al., 1998)

1.3 A promising candidate gene for litter size in pigs is the estrogen receptor gene Independent confirmation of such an effect in several populations might help to confirm the suspected QTL effect of this gene (Rothschild et al., 1996).

The approach for search genes of type (1), known as candidate gene, based on the physiological or biochemical affinity of gene with the nature of the characteristic, has not yet been useful in the identification of an important number of genes in quantitative traits. Its use has been considered with some skepticism in the case of diseases of the mouse (Frankel, 1995).

In the mouse, the number of identified potential markers type (1) is greater than in other species of mammals. In this species, the presence of inbred lines, short generation intervals and reduced costs by individual allow good conditions for detection.

Part of the limitations of the candidate gene approach seems to be the polygenic nature of most traits in animals, the appearance of mutations and the possibility that the supposed QTL is in fact a marker type (2) very next to gene, which allows some probability of recombination. Another possibility is the presence of interactions between genes in different loci (epistasis). These can modify the expression of genes in populations with different genotypes (races or crosses). As an illustration of these difficulties, there are animals detected as free of the porcine malignant hyperthermia mutation with the PCR based test that show the disease (Huges and Lowden, 1998).

\section{Marker-QTL linkage studies}

The second type (2), corresponds to genes linked to the QTL with detectable variation by means of RFLP, microsatellites or other similar molecular systems (Beattie, 1994). Several studies have demonstrated relationships between molecular variants and the phenotypic expression in several characteristics in several animal species (Andersson et al., 1994; A shwell et al., 1997; Beever et al., 1990; Georges et al., 1995; Haley, 1995). This has stimulated the idea to add the genomic to the phenotypic information to increase or speed up the selection response to the "traditional methods" in which it is known as marker-assisted selection or MAS.

In laboratory species, crosses between full inbred lines allow to produce $\mathrm{F} 2$ of segregating backcrosses suitable for efficient detection of QTL-marker linkages. In normal outbreed livestock populations the linkage between the markers and the QTL (linkage disequilibrium) will disappear when is evaluated across families. This makes pointless to compare the average performance of animals with different molecular variants from the whole population to infer marker-QTL associations. Information from crosses of within family analysis is therefore required to detect associations or linkage disequilibrium between the marker and the QTL (Weller et al., 1990).

In livestock, there are basically four design possibilities for marker QTL linkage analysis.

1. Using F2 populations crossing two similar F1 populations, or a backcross between the Fl and one of the original populations.

2. Using a half sib sire design on which heterozygous sires for the markers are mated to a random sample of females and all the progeny is genotyped.

3. Using instead a grandaughter design on which a sire and their sons evaluated by progeny testing are genotyped.

Method 1 allows detecting QTL already fixed in one breed. Methods 2 and 3 are more suitable for prediction of QTL effects for within-population selection.

4. Using crosses of individuals with extreme phenotypes for one trait or trait combination. A nimals from divergently selected lines or from populations with wide variation for important traits are also used.

In outbred populations, only heterozygous sires or grandsires will be informative for the segregation of the marker for the marker and the QTL. In a family, where the marker and the QTL allele are in coupling phase, the effect detected for the substitution of allele $A 1$ of the marker by allele A2 can be of for example $-500 \mathrm{~kg}$ of milk. In another family, where the marker and the QTL allele are in repulsion, the result will be of $+500 \mathrm{~kg}$.

\section{Marker assisted selection (MAS)}

It is currently possible to incorporate this information in the present systems of selection with BLUP, that is to say, to add the information corresponding to the markers to the systems of equations of the mixed model (Goddard, 1992; Kinghorn and Clarke, 1997). 
The identification of the precise position of the QTL in the chromosome, avoids the ambiguity caused by the possibility of recombination between the marker and the QTL. In the later case we should strictly define this kind of selection as QTL assisted selection. For simplicity we treat this option as a special case of MAS without recombination among the marker and the QTL. Current marker use in MAS with the availability of relatively dense genome maps and multiple microsatellite markers available, will be more probably groups of allelic configurations of haplotypes, usually flanking the QTL, than a single marker. These haplotypes could in some cases have very low recombination rates with theQTL.

Several studies of simulation have evaluated the consequences of MAS in populations with a OTL segregating by comparing the use of a purely polygenic animal-BLUP model with a mixed model that incorporates both the polygenic effects and the haplotype identities. An extreme case is the result of Meuwissen and Goddard (1996). They found possible increases until of 30 until a $64 \%$ in the genetic response to selection of different characteristics in the first five generations from selection when the QTL explains a $33 \%$ of the genetic variation in the base population. However, Ruane and Colleau (1995) found possible increases in selection response of only 0.2 to $1 \%$ in six generations using a single marker.

Pongpisantham (1994) found that the indusion of markers could increase up to $15 \%$ the genetic response to selection for growth rate in a population of chickens, compared with selection based on family selection. Ruane and Colleau (1996) found an increase of 6 to $15 \%$ from MAS in the selection response for milk production in cattle nucleus that used multiple ovulation and embryo transfer (MOET) in the first six generations of selection.

In a review, Clarke (1997) concluded that substantial increases to the selection response are possible using MAS in several species. The range of the estimates of increase in selection response for all studies with parameters combinations giving the maximum responses were 2 to $38 \%$. Similar range for parameters combinations giving minimum responses were -0.7 to $22.4 \%$. She conduded that the increases in selection response from MAS could be overestimated by an inadequate modeling of the reduction in the QTL variance by selection.

The variation in the results are a consequence of differences in the assumed sizes of the QTL effects, allelic frequencies, recombination rates between the marker and the QTL, type of marker (single or haplotype), residual polygenic variances, environmental variances, number of generations of selection, population structures and selection procedures. This suggests to maintain an attitude of caution in absence of greater experimental evidence and a greater amount of simulation studies covering more specific situations, that allow to reach certain consensus on the value of the MAS in diverse characters and species.

Meuwissen and Goddard (1996) conduded the following from their study on the use of the MAS:

1. MAS only can increase the rate of genetic gain in the long term, when there is a continuous advantage of new identified QTL (obviously, this has a biological limit determined by the maximum proportion of the genetic variance that can be explained by the segregation of QTL).

2. The extra genetic gain due to the MAS decreases very quickly with the number of generations of selection for a same QTL. The rate of identification of new QTL is difficult to predict.
3. The gain due to MAS for a certain QTL is higher when the characteristic is measured after the selection, as it happens with the fertility and carcass characteristics.

Some studies have shown possible economic advantages of the use of the MAS in dairy cattle (Meuwissen and van Arendonk, 1992; Ruane and Colleau, 1996; Spelman and Garrick, 1997) when the benefits are evaluated for the industry as a whole It is applied with progeny testing or multiple ovulation and embryo transfer (MOET) nuclei improvement schemes.

\section{Other applications of genomic information in livestock improvement}

The increase of the density of genome maps can have other potential beneficial effects in the genetic improvement. Through the identification of paternity in production systems, without controlled mating by means of the use of "genetic fingerprinting" or in the most precise estimation of the genetic relations between the animals, to calculate the additive numerator relationship matrix among animals (A), used in the mixed model equations for the genetic evaluations with BLUP (Nejati-Javaremi et al., 1997).

Mayo (1996) analyzed the possible application of QTL technology to Merino sheep breeding in Australia. He conduded that introduction of new technology will be slow and suggests that the first technology to be used is costeffective DNA parentage, due to the lack of pedigree information, which hinders the utilization of BLUP techniques.

A nother application of genetic fingerprinting is the search of the maximization of dominance effects in crosses (to maximize heterosis) (Godshalk et al., 1990) and their use to control inbreeding. These possible applications are still preliminary ideas that require further evaluation. Current estimation methods of heterosis from crossing experiments are very expensive, thus, indirect prediction of heterosis from genetic diversity at the molecular level may be advantageous.

\section{Marker assisted introgression}

An application that has been mentioned in the literature is the introgression a major gene in another population by means of backcrosses assisted by molecular markers. In this case, it does not seem to exist advantage in using a single genetic marker information, in comparison with the use of only phenotypic information when the characteristic is continuous and the considered genetic effects are additives (Groen and Smith, 1995). Nevertheless, it seems feasible that using a dense map that involves many chromosomal regions and with morethan oneallele of interest, the time for fixation of the major genes can be reduced.

\section{Detection and use of major genes}

In the last ten years statistical methodologies of detection of major genes based on pedigree and phenotypic information on populations have been developed for animal populations. These methods are based on the use of mixed models and segregation analysis to fit the data to a mixture genetic model that indudes in addition to the polygenic effects, those of a biallelic major gene. Calculation is performed in two stages; firstly genotype probabilities are obtained, then major gene, fixed effects and polygenic effects are fitted and used to recalculate new parameters by regressing phenotypes on estimated probabilities. Calculation is iterated upon convergence (Kinghorn et al., 1993). Segregation analysis allows inferring the unknown genotypes from the probabilities of transmission of the gene given the phenotype of the individual and their relatives. 
In mixture models, regression and Gibbs sampling estimation approaches have been implemented to obtain estimates of the major gene effects and allelic frequency (Bovenhuis et al., 1997).

Meuwissen and Goddard (1997) evaluated the effect of induding different proportions of individuals genotyped for a QTL in a mixture model that is based on the analysis of segregation of Kerr and Kinghorn (1996) and a regression approach which uses the estimated genotype probabilities as weights in the estimation process. Unbiased estimates of QTL effect and frequency were obtained in absence of information on the genotype of the QTL, but some improvement in the precision of the estimates were observed as the proportion of genotyped individuals increased. The main limitation of this method is that the genetic hypothesis is generally limited (one biallelic locus), thus, the presence of more alleles could not be detected. Also, the location of the locus in the genome, in absence of markers, remains unknown.

Mixture models can be modified to indude markers associated with the QTL, instead of the direct effect of the QTL in addition to the information of the pedigree and the phenotype. This is achieved by modifying the additive numerator relationship matrix (A), according to the conditional probabilities of transmission of the given QTL the information of the markers (Kinghorn and Clarke, 1997).

These developments can make possible to evaluate the likelihood of the model or another fitting criterion, to prove the relation between the markers and the QTL in populations animals with outbreed mating structures. They also may increase the possibilities of making MAS in animal populations when incomplete information exists on the genotypes of the animals for the QTL or markers so that the use of the genomic information is optimized. Kinghorn (1997a) developed a method to evaluate the amount of genomic information that it allows maximizing a function of economic utility for the analysis of QTL with mixture models.

Major genes have been detected using these methods for carcass characteristics in pigs based on a mixture model of inheritance and Gibbs Sampling (Janss et al., 1995). Also, important effects of major genes have been detected using Findgene software (Kinghorn, 1997b) for several carcass characteristics in cattle (Woodward et al., 1998) and for parasite resistance in sheep (McEwan and Kerr, 1998).

This methods that make use of information currently available in many animal populations, are an option for a preliminary screening for major genes that can contribute to rationalize the use of expensive QTL-marker linkage estimation experiments.

\section{Discussion}

A fundamental aspect for the efficient use of the markers for the MAS resides in the right detection of the location of the QTL in the chromosomes, as well as of the magnitude of its allelic effect and its allelic frequency. Most of the available studies for detection of QTL have limitations of power related to the design, structures of the pedigree and number of animals evaluated for the molecular variants. This causes that, in most of the cases, the estimations have relatively high statistical error. On the other hand, considering that in any study is necessary to accept a value for the error type I (it is to say to reject the hypothesis of existence of the QTL, when this really it does not exist), it is possible that an important fraction of the QTL reported in the literature are a product of the expected number of null hypotheses incorrectly rejected. Low experimental power will reduce the detection rate of true QTL.
The accumulation of studies within the same chromosomal region can help to distinguish between the cases of real or false QTL detection. Meuwissen and Goddard (1996) demonstrated that the incorporation of a false QTL to MAS, can reduce the genetic gain to $14 \%$ in comparison with a usual polygenic BLUP model.

An alternative to determine the value of the MAS, is to use deterministic models of the selection response (Luo et al., 1997). The development of deterministic models that accurately predict the selection response using MAS, could simultaneously optimize selection schemes that involve the selection with BLUP, the use of reproductive techniques like the multiple ovulation and the production in vitro of embryos and the MAS in livestock populations. The use of stochastic simulation, although having advantages, presents as a main difficulty, the required time to obtain a suitable number of replicates. It is also difficult to maximize the response functions due to the number of combinations to study.

Most studies so far have dealt with the effect of MAS using rather simplified assumptions and a single trait affected by one QTL and polygenes. Studies using more real istic models such as multiple estimated QTL effects and multiple trait selection could help to make better decisions regarding the use of MAS in animal improvement.

While molecular techniques offer important options for the genetic improvement of livestock, the materialization of these expectations requires of the solution of a number important of technical problems to take advantage of all the information available an efficient way, to reduce the costs of generating genomic information and to obtain reliable estimations of the effects of the QTL and the application of the MAS and the genomic information in general for the improvement animal. A common problem related with QTL estimates is inconsistency, which means that a QTL effect is not expressed similarly in several years, or when is used in a different population (Mayo and Franklin, 1998).

Many epistatic effects have been found associated to QTL effects. In order to use molecular information in selection, reliable QTL effects should be incorporated in the models of analysis from population genetics in a way, which is consistent with observed variation. Evidences of widespread epistasis affecting QTL effects may restrict use and prediction of QTL effects outside the populations used for detection. This is particularly important when breed crosses instead of sire families in outbreed populations are used as design to detecting the QTL effects (Mayo and Franklin, 1998).

\section{Conclusions}

A rational use of the molecular methodologies requires the simultaneous optimization of selection on all the genes affecting important traits in the population. The maximum benefit can be obtained when these techniques are used in conjunction with reproductive technologies like the artificial insemination, and collection and production in vitro of embryos to accelerate the genetic change (Bishop et al., 1995; Montaldo, 1993). There is a danger associated with a potentially inadequate use of QTL information, giving an excessively high emphasis to simple molecular information in detriment of the overall economic gain through all traits and their polygenic effects in the population. Dissemination of the information to the industry is therefore a complex issue concerning QTL effects and molecular markers.

The characteristics on which the application of the MAS can be effective are those that are expressed late in the life of the animal, or those that are controlled by a few pairs of alleles. The first example corresponds to the longevity and carcass characteristics in meat producing animals; the second, to the resistance to certain diseases or defects of simple inheritance. 
Because of its high cost, the use of MAS could be justified, in animal nudei that allow dilution of the costs when germplasm is extensively used towards the commercial population. Also in those characteristics in which the procedures of conventional selection have reached their imits in efficiency or the results have been not satisfactory.

The main advantage of induding molecular information over pure mixture model and segregation analysis, is the possibility of evaluating the simultaneous effect of several QTL on the characteristics of economic importance, and in the future increasing its precision and the complexity of the involved models of genic action, for example QTL with multiple alleles. The present trend indicates that molecular, pedigree and phenotypic information will be integrated in the future through mixture models of segregation analysis that might contain QTL effects through the markers, polygenic inheritance and the use of powerful and flexible methods of estimation such as Gibbs Sampling.

Before the molecular information on the QTL which control the characteristics of economic interest is generated, the detection of major genes using segregation analysis could direct the work of identification of genotypes towards populations and characteristics with greater probability of detecting a QTL using molecular markers.

Many questions remain on the nature and action of the QTL involved on the variation of complex traits and about the nature and definition of QTL effects. The use of molecular techniques offers new opportunities and challenges for building and using more predictive and efficient statistical models for livestock improvement.

\section{Acknowledgments}

The authors wish to thank Professor Brian Kinghorn and Dr. Julius van Der Werf from the University of New England at Armidale, Australia for inspiring discussions.

\section{References}

Albert, B., Bray, D., Lewis, J., Raff, M., Roberts, K. and Watson, J.' D. (1994). Molecular biology of the cell. Garland Publishing, Inc.

Andersson, L., C.S. Haley, H. Ellegren, S. A. Knott, M. Johansson, K. Andersson, L. Andersson-Eklund, I. EdforsLilja, M. Fredholm, I. Hansson, J. Hakansson, and K. Lundstrom. (1994). Genetic mapping of quantitative trait loci for growth and fatness in the pig. Science 263:1771.

Ashwell, M. S., Rexroad Jr., C.E.,Miller, R.H., Van Raden, P. $\mathrm{M}$. and $\mathrm{Da}, \mathrm{Y}$. (1997). Detection of loci affecting milk production and health traits in an elite US Holstein population using microsatellite markers. Animal Genetics 28:216-222.

Beattie, C. W. (1994). Livestock genome maps. Trends in Genetics 10: 334-338.

Beever, J. E., George, P. D., Fernando, R. L., Stormont, C. J. and Lewin, H. A. (1990). Associations between genetic markers and growth and carcass traits in a parental halfsib familiy of Angus cattle. Journal of A nimal Science 68:337.

Bishop, M. D., Hawkins, G. A . and Keeler, C. L. (1995). Use of DNA markers in animal selection. Theriogenology 43: 61-70.

Bovenhuis, H., van Arendonk, J. A. M., Davis, G., Elsen, J.M., Haley, C. S., Hill, W. G., Baret, P. V., Hetze, D. J. S. and Nicholas, F. W. (1997). Detection and mapping of quantitative trait loci in farm animals. Livestock Production Science 52: 135-144.

Brash, L. D. (1994). Advanced breeding techniques for wool sheep improvement. Wool Technology and Sheep Breeding 42: 327-337.

Buratowski, S. (1994). The basics of basal transcription by RNA polymerasell. Cell 77:1-3.

Chee, M., Yang, R., Hubbell, E., Berno, A., Huang, X. C., Stern, D., Winkler, J. Lockhart, D. J., Morris, M. S. and Fodor, S. P. A. (1996). A ccesing genetic information with highdensity DNA arrays. Science 274:610-614.

Clarke, B. E. (1997). Use of molecular markers in genetic evaluation of animals with applications in Australian Merinos. Ph.D. Thesis. University of New England. A rmidale, NSW, Australia.

Drinkwater, R. D. and Hetzel, D. J. S. (1991). A pplication of molecular biology to understanding genotype-environment interactions in livestock production. In: Proc. of an International Symposium on Nuclear Techniques in Animal Production and Health. IAEA, FAO. Vienna, 15-19 April, 1991: 437-452.

Frankel, W. N. (1995). Taking stock of complex traits in mice. Trends in Genetics 11(12):471-476.

Georges, M., Nielsen, D., Mackinon, M., Mishra, A., Okimoto, R., Pasquino, A. T., Seargeant, L. S. , Sorensen, A, Steele, M. R., Zhao, X., Womack, J. E. and Hoeschele, I. (1995). Mapping quantitative trait loci controlling milk production in dairy cattle by exploiting progeny testing. Genetics 139:907-920.

Georges, M., Grobet, L., Poncelet, D., Royo, L. J., Pirottin, D. and Brouwers, B. (1998). Positional candidate cloning of the bovine $\mathrm{MH}$ locus identifies an allelic series of mutations disrupting the myostatin function and causing doublemuscling in cattle. Proceedings of the 6th Word Congress on Genetics Applied to Livestock Production, Armidale, NSW. Vol. 26: 195-205.

Goddard, M. E. (1992). A mixed model for analyses of data on multiple genetic markers. Theoretical and Applied Genetics 83: 878-886.

Godshalk, E. B., Lee, M. and Lamkey, K. R. (1990). Relationship of restriction fragment length polymorphisms to single-cross hybrid performance of maize Theoretical and A pplied Genetics 80:273-280.

Groen, A. F., and Smith, C. (1995). A stochastic simulation study of the efficiency of marker-assisted introgression in livestock. Journal of A nimal Breeding and Genetics 112:161170.

Haley, C. S. (1995). Livestock QTL - bringing home the bacon?. Trends in Genetics. 11: 488-492.

Henderson, C. R. (1984). Applications of linear models in animal breeding. University of Guelph, Guelp, Canada.

Hoeschele, I., Ulimari, P., Grignola, F. E., Zhang, Q. and Gage, K. M. (1997). Advances in statistical methods to map quantitative trait loci in outbreed populations. Genetics 147: 1445-1457.

Huges, I. and Lowden, S. (1998). A possible genetic basis for false positive hal otane reactions in Australian pigs. Journal of A nimal Breeding and Genetics 115: 113-121.

Janss, L. L. G., Thompson, R., and van Arendonk, J. A. M. (1995). Application of Gibbs sampling for inference in a 
mixed major gene-polygenic inheritance model in animal populations. Theoretical and A pplied Genetics 91: 1137-1147.

Kerr, R. J., and Kinghorn. B. P. (1996). An efficient algorithm for segregation analysis in large populations. Journal of Animal Breeding and Genetics 113: 457-469.

Kinghorn, B. P., (1997a). An index of information content for genotype probabilities derived from segregation analysis. Genetics 145: 479-483.

Kinghorn, B. P., (1997b). FINDGENE analyses at Internet Web site: http://metz.une.edu.au/ bkinghor/findgene.htm

Kinghorn, B. P., Kennedy, B. W. and Smith, C. (1993). A method for screening for genes of major effect. Genetics 134:351-360.

Kinghorn, B. P., van Arendonk, J. A. M. and Hetzel, J. (1994). Detection and use of major genes in animal breeding. AgBiotech News and Information 6(12): 297N-302N .

Kinghorn, B. P., and Clarke, B. E. (1997). Genetic evaluation at individual QTTL. Animal Biotechnology 8: 63-68.

Koleske, A. J., and Young, R. A. (1995). The RNA polymerase holoenzyme and its implications for gene regulation. Trends in Biochemical Sciences 20:113-116.

Lewin, B. (1994). Genes V. Oxford University Press.

Luo, Z. W., Thompson, R., and Woolliams, J. A. (1997). A population genetics model for marker-assisted selection. Genetics 146: 1173-1183.

McEwan, J. C. and Kerr, R. J. (1998). Further evidence that major genes affect host resistance to nematode parasites in Coopworth sheep. In: Proceedings of the $6^{\text {th }}$ Word Congress on Genetics Applied to Livestock Production, Armidale, NSW. Vol. 27:335-338.

Mayo, O. (1996). The application of QTL methodology to Merino breeding. Wool Technology and Sheep Breeding 44: 281-289.

Mayo, O. and Franklin, I. R. (1998). The place of QTL in the basis of quantitative genetics. I. General considerations. In: Proceedings of the $6^{\text {th }}$ Word Congress on Genetics A pplied to Livestock Production, Armidale, NSW. Vol. 26:77-80.

Meuwissen, T. H. E., and Goddard, M. E. (1996). The use of marker-haplotypes in animal breeding schemes. Genetics, Selection, Evolution. 28:161-176.

Meuwissen, T. H. E., and Goddard, M. E. (1997). Estimation of effects of quantitative trait loci in large complex pedigrees Genetics 146:409-940.

Meuwissen, T. H. E., and van Arendonk., J. A. M. (1992). Potential improvements in rate of genetic gain from marker assisted selection in dairy cattle breeding schemes. Journal of Dairy Science 75: 1651-1659.

Montaldo, H. H. (1993). Biotecnología y mejoramiento genético animal en México (Biotechnology and genetic improvement of animals in Mexico). In: (M. Arenas, L. F. Bojalil y $L$. Hernández Comp. Las profesiones en México: Agronomía, Medicina Veterinaria y Zootecnia, Universidad Autónoma Metropolitana-Xochimilco, Universidad de Colima, México, D. F. pp 90-108).

Mullis, K., Facoma, F., Scharf, S., Snikl, R., Horn, G., Erlish, H. (1986). Specific amplification of DNA in vitro: the poymerase chain reaction. Cold Sping Harbor Symposium Quantitative Biology 51:260.
Nejati-Javaremi, A., Smith, C. and Gibson, J. P. (1997). Effect of total allelic relationship on accuracy of evaluation and response to selection. Journal of A nimal Science 75:1738-1745.

Pongpisantham, B. (1994). A pplying genotype and markerassisted selection for the improvement of quantitative traits in poultry. M.S. thesis. University of New England, pp. 121.

Rotshshild, M., Jacobson, C., Vaske, D. Tuggle, C., Wang, L., Short, T., Eckard, G., Sasaki, S., Vincent, A., McLaren, D., Southwood, O., van der Steen, H., Mileham, A. and Plastow, G. (1996). The estrogen receptor locus is associated with a major gene influencing litter size in pigs. In: Proceeding of the National A cademy of Sciences USA 93:201-205.

Ruane, J. and Colleau, J. J. (1995). Marker assisted selection for genetic improvement of animal populations when a singleQTL is marked. Genet. Res. Camb. 66:71-83.

Ruane, J., and Colleau, J. J. (1996). Marker assisted selection for a sex-limited character in a nucleus breeding population. Journal of Dairy Science 79 : 1666-1678.

Sax, K. (1923). The association of size differences with seedcoat pattern and pigmentation in Phaseolus vulgaris Genetics 8: 522-560.

Schwerin, M., Brockmann, G., Vanselow, J., and Seyfert, H. M. (1995). Perspectives of molecular genome analysis in livestock improvement. A rch. Tierz. Dummerstorf 38: 21-31.

Smith, C., and Smith, D. B. (1993). The need for close limkages in marked-assisted selection for economic merit in livestock. Animal Breeding A bstracts 61: 197-204.

Spelman, R. and Garrick, D. (1997). Utilisation of marker assisted selection in a commercial dairy cow population. Livestock Production Science 47:139-147.

Stein, G. S., Stain, J. L., van Wijnen, A. J. and Lian, J. B. (1996). The maturation of a cell. American Scientist 84: 28-37.

Schimenti, J. (1998). Global analysis of gene function in mammals: integration of physical, mutational and expression strategies. EJB Electronic Journal of Biotechnology at http://ejb.ucv.cl/content/vol1/issue1/full/5/.

Weller, J. I., Kashi, Y. and Soller, M. (1990). Power of daughter and granddaughter designs for determining linkage between marker loci and quantitative trit loci in dairy cattle. Journal of Dairy Science 73: 2525-2537.

Wilmut, I., Haley, C. S., Simons, J. P. and Webb, R. (1992). The potential role of moelcular genetic manipulation in the improvement of reproductive performance Journal of Reproduction and Fertility. Supplement 45: 157-173.

Woodward, B. W., Du, F.-X., Montaldo, H., Andersen, J. and DeNise, S. K. (1998).Preliminary evidence for major genes controlling beef carcass traits in Limousin cattle. In: Proc. of the $6^{\text {th }}$ Word Congress on Genetics Applied to Livestock Production, Armidale, NSW. Vol. 25: 157-160. 